\title{
Hemorrhagic Thoracic Spinal Epidural Angiolipoma: A Case Report
}

\author{
Saufi $\mathrm{A}^{\mathrm{a}}$, Chan $\mathrm{KH}^{\mathrm{a}}$, Zamzuri $\mathrm{Z}^{\mathrm{b}}$, Ralib AR ${ }^{\mathrm{c}}$, Azlina $\mathrm{AR}^{\mathrm{d}}$ \\ aUnit of Neurosurgery, Department of Surgery, International Islamic University Malaysia, Malaysia \\ bDepartment of Orthopedic, International Islamic University Malaysia, Malaysia \\ 'Department of Radiology, International Islamic University Malaysia, Malaysia \\ dDepartment of Pathology, Hospital Tengku Ampuan Afzan, Malaysia
}

\section{ABSTRACT}

Spinal epidural angiolipomas are rare benign tumors. The tumors contain both vascular and mature adipose components. Typically, they present with a progressive clinical course. Acute presentation is rare. We present a case of a fifteen-year-old girl who presented with a sudden onset of backpain followed by paraparesis with bladder and bowel incontinence. Neurological examination showed sensory loss below T10 with Grade 2 motor power. Spinal MRI showed long segment extradural lesion at T6-T9 level. Multiple laminectomy and excision of the tumor containing areas of hemorrhage was performed. Histopathology report revealed angiolipoma.

KEYWORDS: Angiolipoma, cord compression, epidural spinal tumor, spinal epidural hematoma

\section{INTRODUCTION}

Spinal epidural angiolipomas are rare benign tumors composed of mature lipocytes with abnormal blood vessels. They account for $0.14-1.2 \%$ of tumors of the spinal axis and approximately $3 \%$ of epidural spinal tumors. ${ }^{1,2}$ Spinal epidural angiolipoma can exist in two subtypes: infiltrating and non- infiltrating with the latter much more common. ${ }^{2}$ They generally present with progressive spinal cord compression. According to Akhaddar et al (2008) ${ }^{1}$, acute presentation is rare.

We present a case report of an intraspinal hemorrhage from the thoracic epidural angiolipoma presented with acute paraparesis.

\section{CASE REPORT}

A 15-year-old girl presented with an acute onset of back pain while walking followed by paraparesis. During the hospital admission, she developed urinary and bowel incontinence. There was no history of trauma or bleeding disorder. Physical examination revealed paraparesis Grade 2 muscle power with hypotonia and hyporeflexia, and sensory loss from T10 downwards. A

Corresponding author:

Assoc. Prof. Dr. Mohamed Saufi B. Awang

Department of Surgery

Kulliyyah of Medicine

International Islamic University Malaysia

25200 Kuantan, Pahang

Email: saufiawang@yahoo.com

Tel: 09-5706171

HIP: 012-908 3420 thoracic spine MRI revealed a long segment extradural lesion at T6-T9 level (Figure 1). Multiple levels laminectomy and surgical removal of the lesion was performed.
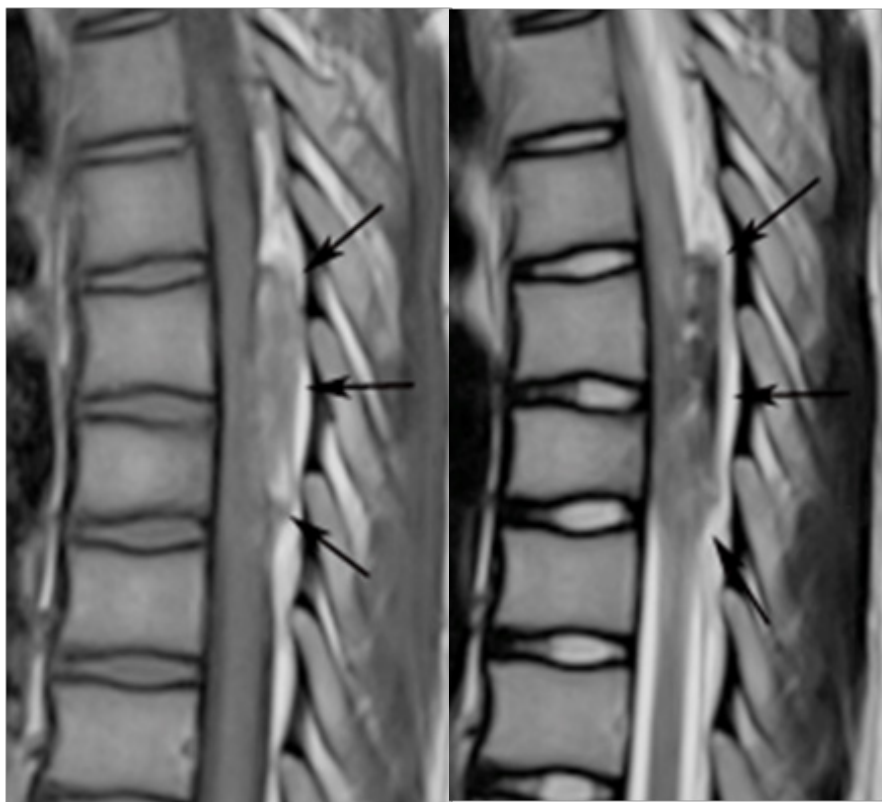

Figure 1. MRI of the thoracic spine in sagital T1 and sagital T2 sequences showing an elongated oval shaped intradural extramedullary mass (black arrows) located posterolateral to the thoracic spinal cord. The main component of the mass is isointense to the spinal cord on T1. Within the mass there are heterogenous areas, which appear hyperintense on $\mathrm{T} 1$ and mixed intensities on T2, suggestive of various stages of blood products. No significant enhancement seen post IV gadolinium (not shown). 
Intraoperatively, there was well formed epidural hematoma with cylinder elongated solid lesion within it. Histopathological examination revealed angiolipoma (Figure 2). Postoperatively, she made a good recovery.
She was able to ambulate within three months. Her bladder and bowel functions returned to normal. She has been well for more than a year of follow-up. Post operative MRI at six months showed no residual tumor.

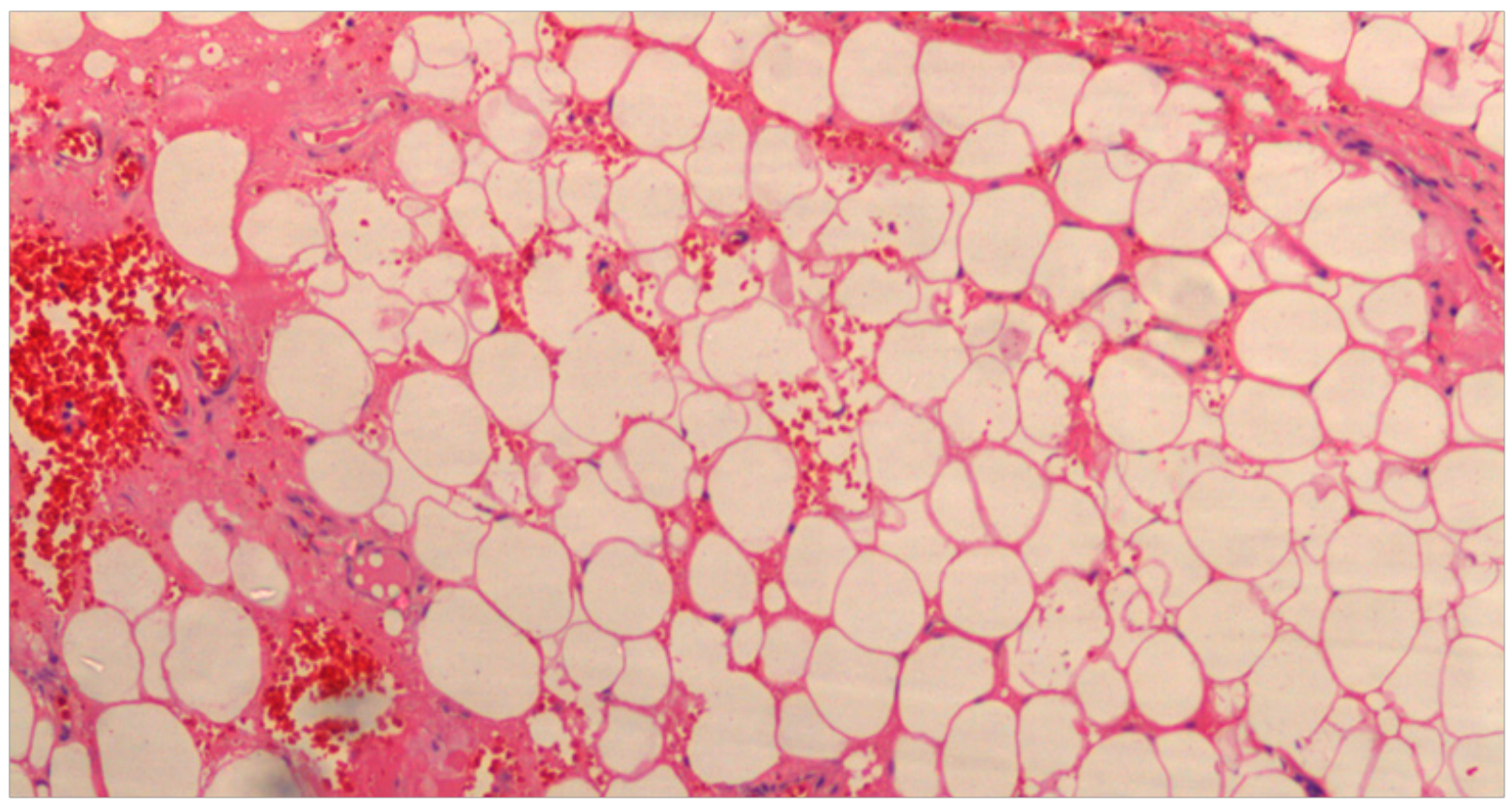

Figure 2. Showing lobules of mature adipocytes with foci of haemorrhage and many scattered small vessels. (Magnification x200)

\section{DISCUSSION}

Spinal angiolipomas are rare benign tumors that account for $0.14 \%$ of all spinal axis tumor. ${ }^{1,2,3}$ The first case of spinal angiolipoma was described by Berenbruch in 1890.2 Spinal angiolipoma usually produce a progressive symptom. However, there have been reports that spinal angiolipoma may present with remitting and relapsing course and to some extent, acute or subacute presentation, which is exceedingly rare. $^{1}$

There are various factors, which may contribute to the development of acute onset of symptoms. These include rapid expansion of tumor volume caused by hemorrhage or enlarging blood vessels. Other factors like extracellular fluid volume change, pregnancy and alteration in fat mobilization pattern may cause to an increase in tumor volume. ${ }^{1,4}$ In this case, the rapid onset was due to hemorrhage. However, no obvious risk factors leading to this were identified in this patient.

MRI is still the imaging of choice in detecting the spinal angiolipoma. . $^{2,3,4}$ Typically, MRI demonstrates angiolipoma as a fatty mass with an abundant vascular component. On T1-weighted image, the mass usually appears to be iso-hyperintense and inhomogeneous hyperintense on T2-weighted image. Heterogenous hyperintensity to spinal cord with focal hypointensity on T2-weighted image is suggestive of acute or hyperacute epidural hematoma. ${ }^{1}$
Pathologically, spinal angiolipoma is composed of mature fat cells abundant with small blood vessels. The mass is divided into lobules by the thin fibrous capsules with fibrous septa. The diagnostic feature is characterized by the presence of fibrinous microthrombi in the lumen. ${ }^{3,5}$

The mainstay of treatment is complete excision of the tumor and evacuation of the epidural hematoma. Early surgery is highly recommended as the prognosis depends not only on the degree of neurologic dysfunction at presentation but more importantly is the time from the symptom onset to surgical decompression..$^{1,3,4}$ As illustrated here, the patient showed good recovery following early decompression and excision of the tumor.

Usually non-infiltrating epidural angiolipomas can be resected completely in contrast to infiltrating angilipomas. ${ }^{4,5}$ The role of radiotherapy is controversial. However, there were reports that postoperative radiation was given to patients with partial excision. ${ }^{6}$ Overall prognosis is good even with partial surgical resection as angiolipomas are benign tumors. Therefore, postoperative radiation should be given with caution in order to avoid unnecessary complications. 


\section{DISCUSSION}

Spinal angiolipomas are rare benign tumors that account for $0.14 \%$ of all spinal axis tumor. ${ }^{1,2,3}$ The first case of spinal angiolipoma was described by Berenbruch in 1890. ${ }^{2}$ Spinal angiolipoma usually produce a progressive symptom. However, there have been reports that spinal angiolipoma may present with remitting and relapsing course and to some extent, acute or subacute presentation, which is exceedingly rare. $^{1}$

There are various factors, which may contribute to the development of acute onset of symptoms. These include rapid expansion of tumor volume caused by hemorrhage or enlarging blood vessels. Other factors like extracellular fluid volume change, pregnancy and alteration in fat mobilization pattern may cause to an increase in tumor volume. ${ }^{1,4}$ In this case, the rapid onset was due to hemorrhage. However, no obvious risk factors leading to this were identified in this patient.

MRI is still the imaging of choice in detecting the spinal angiolipoma. . $^{2,3}$ Typically, MRI demonstrates angiolipoma as a fatty mass with an abundant vascular component. On T1-weighted image, the mass usually appears to be iso-hyperintense and inhomogeneous hyperintense on T2-weighted image. Heterogenous hyperintensity to spinal cord with focal hypointensity on T2-weighted image is suggestive of acute or hyperacute epidural hematoma. ${ }^{1}$

Pathologically, spinal angiolipoma is composed of mature fat cells abundant with small blood vessels. The mass is divided into lobules by the thin fibrous capsules with fibrous septa. The diagnostic feature is characterized by the presence of fibrinous microthrombi in the lumen. ${ }^{3,5}$

The mainstay of treatment is complete excision of the tumor and evacuation of the epidural hematoma. Early surgery is highly recommended as the prognosis depends not only on the degree of neurologic dysfunction at presentation but more importantly is the time from the symptom onset to surgical decompression..$^{1,3,4}$ As illustrated here, the patient showed good recovery following early decompression and excision of the tumor.

Usually non-infiltrating epidural angiolipomas can be resected completely in contrast to infiltrating angilipomas. ${ }^{4,5}$ The role of radiotherapy is controversial. However, there were reports that postoperative radiation was given to patients with partial excision. ${ }^{6}$ Overall prognosis is good even with partial surgical resection as angiolipomas are benign tumors. Therefore, postoperative radiation should be given with caution in order to avoid unnecessary complications.

\section{CONCLUSION}

Spinal epidural angiolipoma is a rare benign tumor that usually presents with progressive symptoms. In some occasions, it can present in an atypical manner as illustrated here. MRI is usually diagnostic. Early detection and rapid surgical intervention are recommended as the prognosis is favourable with early treatment.

\section{REFERENCES}

1. Akhaddar A, Albouzidi A, Elmostarchid B, Gazzaz $M$, Boucetta $M$. Sudden onset of paraplegia caused by hemorrhagic spinal epidural angiolipoma. A case report. Eur Spine J 2008; 17:S296-8.

2. Rocchi G, Caroli E, Frati A, Cimatti M, Savlati. Lumbar spinal angiolipomas: report of two cases and review of the literature. Spinal Cord 2004; 42:313-6.

3. Koul R, Dubey A. Spinal Angiolipoma. J HK Coll Radiol 2009; 12:20-3.

4. Samdani AF, Garonzik IM, Jallo G, Eberhart G, Zhos. Spinal angiolipomas: case report and review of the literature. Acta Neurochir 2004; 146:299302.

5. Haddad FS, Abla A, Allam CK. Extradural Spinal Angiolipoma. Surg Neurol 1986; 26:473-86.

6. Fourney DR, Griebel RW, Tong KA, et al. Spinal angiolipoma. Can J Neurol Sci 2001; 28:82-8. 
